\title{
Pushing the boundaries: transesophageal endoscopic ultrasound-guided fine-needle aspiration for the diagnosis of intraparenchymal pulmonary micronodules
}

\author{
Stefano Maria Donghi ${ }^{1}$, Elena Prisciandaro ${ }^{1}$, Niccolò Filippi $^{1}$, Juliana Guarize ${ }^{1}$, Lorenzo Spaggiari ${ }^{1,2}$ \\ ${ }^{1}$ Division of Thoracic Surgery, IEO, European Institute of Oncology IRCCS, Milan, Italy; ${ }^{2}$ Division of Oncology and Hemato-Oncology, University \\ of Milan, Milan, Italy \\ Contributions: (I) Conception and design: SM Donghi; (II) Administrative support: L Spaggiari; (III) Provision of study materials or patients: J \\ Guarize; (IV) Collection and assembly of data: N Filippi; (V) Data analysis and interpretation: E Prisciandaro; (VI) Manuscript writing: All authors; \\ (VII) Final approval of manuscript: All authors. \\ Correspondence to: Stefano Maria Donghi, MD. Division of Thoracic Surgery, IEO, European Institute of Oncology - Via Ripamonti, 435,20141 \\ Milan, Italy. Email: stefanomaria.donghi@ieo.it.
}

\begin{abstract}
Endobronchial ultrasonography-guided transbronchial needle aspiration (EBUS-TBNA) is nowadays considered widely reliable for mediastinal lymph nodal staging in lung cancer patients. In a few years it has deeply changed the diagnostic algorithms for thoracic diseases and can guide the choice of therapeutic protocols. The use of an EBUS videobronchoscope for transesophageal exploration (EUS-B-FNA) is less conventional. Here we report the case of a patient who underwent transesophageal EUS-B-FNA of a periesophageal pulmonary nodule in order to confirm systemic recurrence of a tracheal tumor. EUS-B-FNA is a reliable alternative technique for the diagnosis of pulmonary lesions, being effective and well tolerated by patients. The versatility of the EBUS bronchoscope gives reason, among others, for the key role it plays in the management of lung cancer patients.
\end{abstract}

Keywords: Lung nodules; tracheal tumor; adenoid-cystic carcinoma; transesophageal endobronchial ultrasonography (transesophageal EBUS)

Received: 05 September 2019; Accepted: 28 November 2019; Published: 10 April 2020.

doi: $10.21037 /$ shc.2019.12.01

View this article at: http://dx.doi.org/10.21037/shc.2019.12.01

\section{Introduction}

Lung cancer is the leading cause of tumor-related death worldwide (1), representing a major global-health issue. Recent advances in lung cancer treatment highlight the need for pathological specimens for diagnosis, staging and molecular testing. On the other hand, technological innovation has provided valid alternatives to surgical biopsies: minimally invasive procedures are safer, better tolerated by patients and non-inferior in terms of efficacy. Endobronchial ultrasonography-guided transbronchial needle aspiration (EBUS-TBNA) is nowadays considered widely reliable for mediastinal lymph nodal staging in lung cancer patients. In a few years it has deeply changed the diagnostic algorithms for thoracic diseases and can guide the choice of therapeutic protocols (2). In addition, EBUSTBNA also finds a role in the investigation of a large variety of lesions close to the main airways, e.g. thyroid and lung nodules (3), pleural thickenings (4), mediastinal masses. Moreover, the EBUS bronchoscope can be employed for ultrasonographic evaluation of the lungs and mediastinum through the esophageal walls, allowing the detection of periesophageal lesions and their sampling by means of transesophageal fine needle aspiration (FNA).

Here we report the case of a patient who underwent transesophageal endoscopic ultrasound-guided fine-needle aspiration (EUS-B-FNA) of a periesophageal pulmonary nodule in order to confirm systemic recurrence of a tracheal 


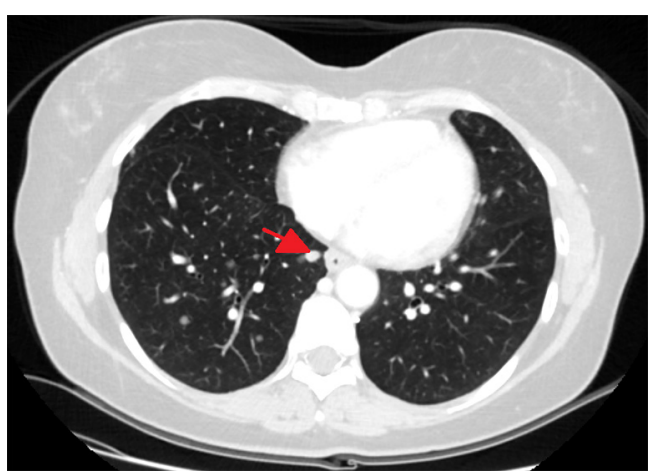

Figure 1 Chest CT (axial view) showing an infracentimetric pulmonary nodule in the right lower lobe, adjacent to the distal tract of the esophagus (arrow).

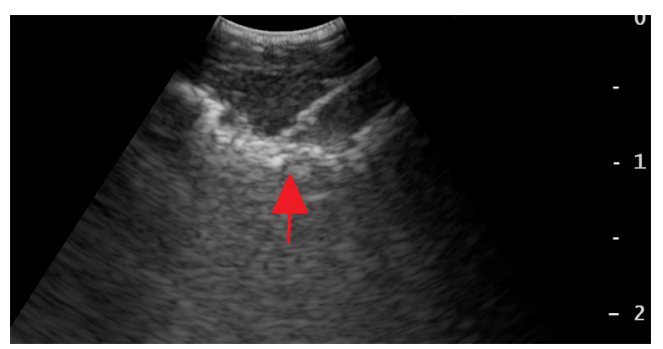

Figure 2 EUS-B-FNA of the periesophageal nodule (arrow), appearing at ultrasonographic evaluation as a round, welldefined and hypoechoic lesion. EUS-B-FNA, endobronchial ultrasonography-guided transbronchial needle aspiration.

tumor. The case is presented in accordance with the CARE Guidelines.

\section{Case presentation}

A 50-year-old woman presented with multiple bilateral pulmonary micronodules discovered on chest computed tomography (CT). She had a history of tracheal adenoidcystic carcinoma and she had undergone carinal resection and reconstruction 3 years earlier. Follow-up CT scans revealed multiple bilateral infracentimetric nodules, randomly located throughout the pulmonary parenchyma. ${ }^{18}$ F-Fluoro-deoxy-glucose positron emission tomography $\left({ }^{18} \mathrm{~F}-\mathrm{FDG} \mathrm{PET}\right)$ showed an increased uptake in all the lung nodules, in absence of any other hypermetabolic lesions. Flexible fiberoptic videobronchoscopy did not find any tracheobronchial lesions and showed a healthy tracheal anastomosis. In order to confirm or rule out the suspicion of tracheal cancer recurrence, pathological specimens were needed. The parenchymal nodules not being adjacent to the main airways, EBUS-TBNA could not be carried out. Having undergone previous chest surgery by longitudinal median sternotomy, thoracoscopic biopsies of the pulmonary nodules were not considered the best diagnostic option. Instead, transesophageal EUS-B-FNA under conscious sedation was performed on an infracentimetric periesophageal lung nodule in the right lower lobe (Figure 1). The lesion was reached through the right posterior-lateral wall of the distal esophagus. At ultrasonographic evaluation, the nodule appeared as a round, well-defined and hypoechoic lesion (Figure 2). Pathological analysis on EUS-B-FNA samples was suggestive of tracheal adenoid-cystic carcinoma. No adverse events occurred during and immediately after the procedure. The patient is currently on chemotherapy.

\section{Discussion}

EBUS-TBNA is a fundamental tool for the diagnosis, staging and molecular testing of lung cancer and it has gained importance in everyday clinical practice of pulmonologists and thoracic surgeons. This technique is usually employed to target mediastinal lymph nodes, masses or pulmonary nodules close to the hilum, but it potentially allows to sample all kinds of lesions contiguous to the main airway.

The use of an EBUS videobronchoscope for transesophageal exploration (EUS-B-FNA) is less conventional. By inserting the instrument into the esophagus, an ultrasonographic evaluation of the adjacent anatomical structures can be carried out through the esophageal walls. This peculiar procedure has already been described $(5,6)$ with excellent results in terms of safety and diagnostic yield. In addition, the reported complication rate is very low and it can be carried out during the same endoscopy session as EBUS-TBNA by one operator.

In the reported case, a CT-guided fine needle aspiration biopsy (FNAB) could not be performed, due to the absence of peripheral pulmonary lesions. EBUS failed to reveal any lung nodule that could be sampled through the tracheal or bronchial walls. On the other hand, in order to prevent the patient from undergoing a second thoracic surgery, thoracoscopic lung biopsies were not recommended. EUSB-FNA represented a valid solution to this challenging 
diagnostic issue. There were no peri- or post-operative complications and the patient was dismissed after of few hours of clinical observation.

In conclusion, EUS-B-FNA is a reliable alternative technique for the diagnosis of pulmonary lesions, being effective and well tolerated by patients. The versatility of the EBUS bronchoscope gives reason, among others, for the key role it plays in the management of lung cancer patients.

\section{Acknowledgments}

The authors would like to thank Simona Prisciandaro for proofreading the text.

Funding: None.

\section{Footnote}

Provenance and Peer Review: This article was commissioned by the Guest Editors (Giulia Sedda and Roberto Gasparri) for the series "A New Era in Lung Cancer Care: from Early Diagnosis to Personalized Treatment" published in Shanghai Chest. The article has undergone external peer review.

Conflicts of Interest: All authors have completed the ICMJE uniform disclosure form (available at http://dx.doi. org/10.21037/shc.2019.12.01). The series “A New Era in Lung Cancer Care: from Early Diagnosis to Personalized Treatment" was commissioned by the editorial office without any funding or sponsorship. LS serves as an unpaid editorial board member of Shanghai Chest from Aug 2019 to Jul 2021. The authors have no other conflicts of interest to declare.

Ethical Statement: The authors are accountable for all aspects of the work in ensuring that questions related to the accuracy or integrity of any part of the work are appropriately investigated and resolved. All procedures performed in studies involving human participants were in accordance with the ethical standards of the institutional and/or national research committee(s) and with the Helsinki Declaration (as revised in 2013). Written informed consent was obtained from the patient for publication of this Case Report and any accompanying images. A copy of the written consent is available for review by the Editor-in- Chief of this journal.
Open Access Statement: This is an Open Access article distributed in accordance with the Creative Commons Attribution-NonCommercial-NoDerivs 4.0 International License (CC BY-NC-ND 4.0), which permits the noncommercial replication and distribution of the article with the strict proviso that no changes or edits are made and the original work is properly cited (including links to both the formal publication through the relevant DOI and the license). See: https://creativecommons.org/licenses/by-nc$\mathrm{nd} / 4.0 \%$.

\section{References}

1. Fitzmaurice C, Allen C, Barber RM, et al. Global, Regional, and National Cancer Incidence, Mortality, Years of Life Lost, Years Lived With Disability, and DisabilityAdjusted Life-years for 32 Cancer Groups, 1990 to 2015: A Systematic Analysis for the Global Burden of Disease Study. JAMA Oncol 2017;3:524-48.

2. Guarize J, Casiraghi M, Donghi S, et al. Endobronchial Ultrasound Transbronchial Needle Aspiration in Thoracic Diseases: Much More than Mediastinal Staging. Can Respir J 2018;2018:4269798.

3. Filippi N, Prisciandaro E, Guarize J, et al. One-shot diagnosis: EBUS-TBNA as a single procedure for thyroid, pulmonary and lymph nodal lesions. Adv Respir Med 2019;87:194-5.

4. Donghi SM, Prisciandaro E, Sedda G, et al. When Less Is More: EBUS-TBNA for the Diagnosis of Pleural Lesions. Innovations (Phila) 2019;14:473-5.

5. Steinfort DP, Farmer MW, Irving LB, et al. Pulmonologist-Performed Per-Esophageal Needle Aspiration of Parenchymal Lung Lesions Using an EBUS Bronchoscope: Diagnostic Utility and Safety. J Bronchology Interv Pulmonol 2017;24:117-24.

6. Skovgaard Christiansen I, Kuijvenhoven JC, Bodtger U, et al. Endoscopic Ultrasound with Bronchoscope-Guided Fine Needle Aspiration for the Diagnosis of Paraesophageally Located Lung Lesions. Respiration 2019;97:277-83.

doi: $10.21037 /$ shc.2019.12.01

Cite this article as: Donghi SM, Prisciandaro E, Filippi N, Guarize J, Spaggiari L. Pushing the boundaries: transesophageal endoscopic ultrasound-guided fine-needle aspiration for the diagnosis of intraparenchymal pulmonary micronodules. Shanghai Chest 2020;4:11. 